\title{
Estimation of Vehicle State and Road Coefficient for Electric Vehicle through Extended Kalman Filter and RLS Approaches
}

\author{
LIN Cheng ${ }^{1}$, WANG Gang ${ }^{1, a}$, CAO Wan-ke ${ }^{1}$ and ZHOU Feng-jun ${ }^{1, b}$ \\ ${ }^{1}$ The National Engineering Laboratory for Electric Vehicles, Beijing Institute of Technology, China

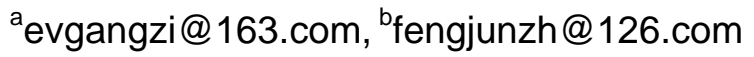

Keywords: Electric vehicle, extended Kalman filter (EKF), estimation of vehicle state and road coefficient, recursive least squares (RLS).

\begin{abstract}
Estimation of vehicle state (e.g., vehicle velocity and sideslip angle) and road friction coefficient is essential for electric vehicle stability control. This article proposes a novel real-time model-based vehicle estimator, which can be used for estimation of vehicle state and road friction coefficient for the distributed driven electric vehicle. The estimator is realized using the extended Kalman filter (EKF) and the recursive least squares (RLS) technique. The proposed estimation algorithm is evaluated through simulation and experimental test. Results to data indicate that the proposed approach is effective and it has the ability to provide with reliable information for vehicle active safety control.
\end{abstract}

\section{Introduction}

Active safety technologies include anti-lock braking system, traction control system and stability control system. They can prevent the risky situation which may require the information about the vehicle dynamic states as well as road condition. ${ }^{[1-2]}$ Due to the technical infeasibility and economic reason, some states are immeasurable in real time. They are required to be obtained by estimation. This has spurred a lot of research activity devoted to the estimation, resulting in many kinds of algorithm to estimate the vehicle dynamic states and the road condition ${ }^{[3-4]}$.

The electric vehicle (EV) is driven by electric motor. The Real-time motor torque and speed can be transmitting to the controller as feedback. The wheel's driving force can be calculated based on the above information ${ }^{[5]}$. This article aims to develop a real-time estimation of vehicle state and road surface based on the distributed driven electric vehicle. In vehicle state estimation, extended Kalman filter algorithm is utilized based on a four-wheel vehicle model and the information obtained from the sensors and CAN-BUS. In road friction estimation, a recursive least square algorithm is utilized. Simulation results, obtained with the CarSim program demonstrate a good performance of the EKF algorithm. The test results show that the RLS algorithm is able to accurately identify road surface.

\section{Vehicle model}

\section{Nonlinear vehicle model ${ }^{[6]}$}

Ignoring air resistance and slope angle, a nonlinear model with four wheels is selected. This model is used to estimate the vehicle dynamic state. The model is presented in Figure 1.

The equations of the motion nonlinear four tires vehicle dynamic model are as follows:

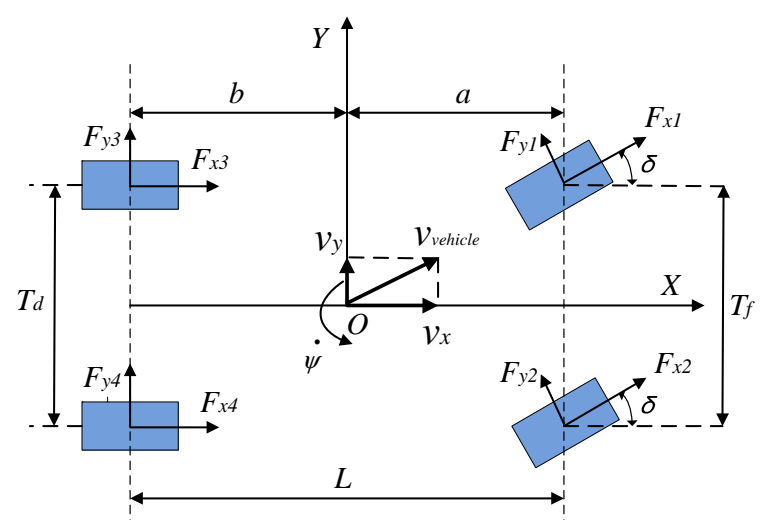

Figure.1 Four tires vehicle dynamic model 


$$
\left\{\begin{aligned}
\dot{V}_{x}= & 1 / m \cdot\left(\left(F_{x 1}+F_{x 2}\right) \cdot \cos \delta-\left(F_{y 1}+F_{y 2}\right) \cdot \sin \delta+\left(F_{x 3}+F_{x 4}\right) \cdot \cos \beta+\left(F_{y 3}+F_{y 4}\right) \cdot \sin \beta\right)+V_{y} \cdot \dot{\psi} \\
\dot{V}_{y}= & 1 / m \cdot\left(\left(F_{y 1}+F_{y 2}\right) \cdot \cos \delta+\left(F_{y 3}+F_{y 4}\right) \cdot \cos \beta+\left(F_{x 1}+F_{x 2}\right) \cdot \sin \delta-\left(F_{x 3}+F_{x 4}\right) \cdot \sin \beta\right)-V_{x} \cdot \dot{\psi} \\
\ddot{\psi}= & 1 / I_{z} \cdot\left(a \cdot\left(F_{x 1}+F_{x 2}\right) \cdot \sin \delta+a \cdot\left(F_{y 1}+F_{y 2}\right) \cdot \cos \delta-b \cdot\left(F_{y 3}+F_{y 4}\right)+0.5 \cdot t_{f} \cdot\left(F_{x 2}-F_{x 1}\right) \cdot \cos \delta\right. \\
& \left.+0.5 \cdot t_{d} \cdot\left(F_{x 4}-F_{x 3}\right)-0.5 \cdot t_{f} \cdot\left(F_{y 2}-F_{y 1}\right) \cdot \sin \delta\right)
\end{aligned}\right.
$$

Longitudinal and vertical forces at each tire are as follows:

$$
\left\{\begin{array}{l}
F_{z 1,2}=\left(0.5 \cdot m \cdot g \mp m \cdot a_{y} \cdot h / t_{d}\right) \cdot b / L-0.5 \cdot m \cdot a_{x} \cdot h / L \\
F_{z 3,4}=\left(0.5 \cdot m \cdot g \mp m \cdot a_{y} \cdot h / t_{d}\right) \cdot a / L+0.5 \cdot m \cdot a_{x} \cdot h / L \\
F_{x 1,2}=1 / r \cdot\left(T_{x 1,2}-J \cdot \omega_{x 1,2}\right) \\
\beta=\arctan \left(V_{y} / V_{x}\right) \\
\mu_{i}=\frac{F_{x i}}{F_{z i}}(i=1,2,3,4)
\end{array}\right.
$$

where $\omega_{\text {is }}$ the angular speed of the wheel, $T_{i}$ is the driving torque, $F_{x}$ is the longitudinal tire-road contact force, $F_{y}$ is the lateral tire-road contact force, $F_{z}$ is the vertical tire-road contact force, $r$ is the wheel radius, $\mu$ is the road friction coefficient. $b$ is the distance between cog and rear axle, ${ }^{h}$ is the height of the vehicle center of gravity, $a$ is the distance between front axle and front axle.

\section{Semi-empirical tire model ${ }^{[6]}$}

As for the road friction vector $\mu$, we employ a widely used model known as the Burckhardt model.

$$
\mu(\lambda)=C_{1}\left(1-e^{-\lambda C_{2}}\right)-C_{3} \lambda
$$

The value $C_{2}$ of the equation (3) cannot be recognized using the below method. The improved tire formula as follow:

$$
\mu(\lambda)=C_{1}-A_{1} e^{-23.99 \lambda}-A_{2} e^{-33.82 \lambda}-A_{3} e^{-94.13 \lambda}-A_{4} e^{-306.39 \lambda}-C_{3} \lambda
$$

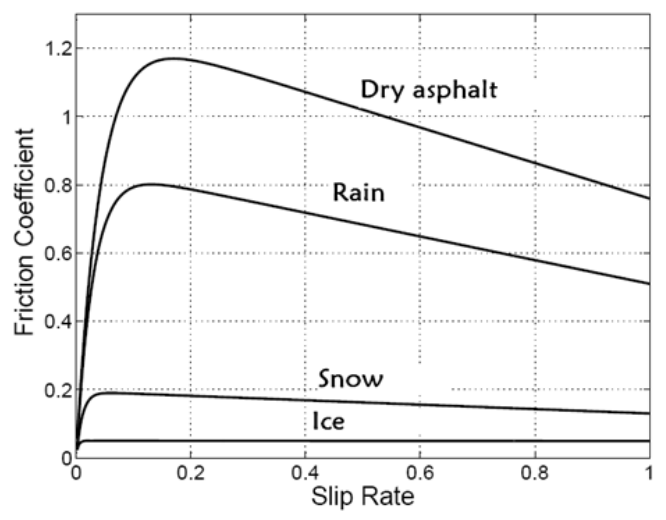

Figure.2 The friction coefficient in different road condition

\section{Vehicle state estimation and road friction estimation}

\section{Vehicle state estimation}

The EKF algorithm is a powerful extension of the standard Kalman filter ${ }^{[7]}$.It is designed as estimation for non-linear system. The vehicle state estimator in this article is based on the EKF. The nonlinear discrete-time system as follow:

$$
\left\{\begin{array}{l}
x_{k+1}=f\left(x_{k}, u_{k}, w_{k}\right) \\
Z_{k}=h\left(x_{k}, v_{k}\right)
\end{array}\right.
$$

The nonlinear vehicle model can be transformed into standard state-space form.

State vector: $x=\left[\begin{array}{lllllll}V_{x} & V_{y} & \dot{\psi} & F_{x i} & F_{z i} & \beta & \mu_{i}\end{array}\right]_{(i=1,2,3,4)}^{T}$

Input vector: $u=\left[\begin{array}{ll}\delta & \omega_{\mathrm{i}}\end{array}\right]_{(i=1,2,3,4)}$

\section{Road friction estimation}

Least squares identification algorithm is a classical estimation method. It has a wide range of 
application. However, it is unsuitable for online identification ${ }^{[8,9]}$. The recursive least squares algorithm can reduce the amount of computation and memory usage. It has better instant communication. In this paper, this algorithm is as the road identification method ${ }^{[10]}$.

When vehicle is traveling on varied roads, the slip-adhesion coefficient curves are different. Assuming that the slip-friction coefficient curves of the four kind's road (dry asphalt, rain, snow, ice) are available. When the vehicle is traveling on the road, which kind of road conditions is unknown. According to the above equation (3) we can obtain the slip-friction coefficient curve of this kind of road. The Burckhardt model is nonlinear because of the $C_{2}$. As an alternative, the improved Burckhardt model is approximated using a linear regression. The RLS methods can be directly applied to test against four different friction curves. The type of road condition can be identified.

\section{Real vehicle experimental tests}

\section{Experimental equipment and experimental methods}

The test car used for the experiments described in this work is a distributed driven electric vehicle. Test drives have been carried out on two different road conditions, i.e. dry asphalt and ice-road. When the vehicle travelling on the straight line, the state vectors were estimated by EKF algorithm. The curve map of tire slipping rate-road coefficient could be draw up. The above estimation algorithm was used to identify the road condition and the maximum road coefficient was estimated.
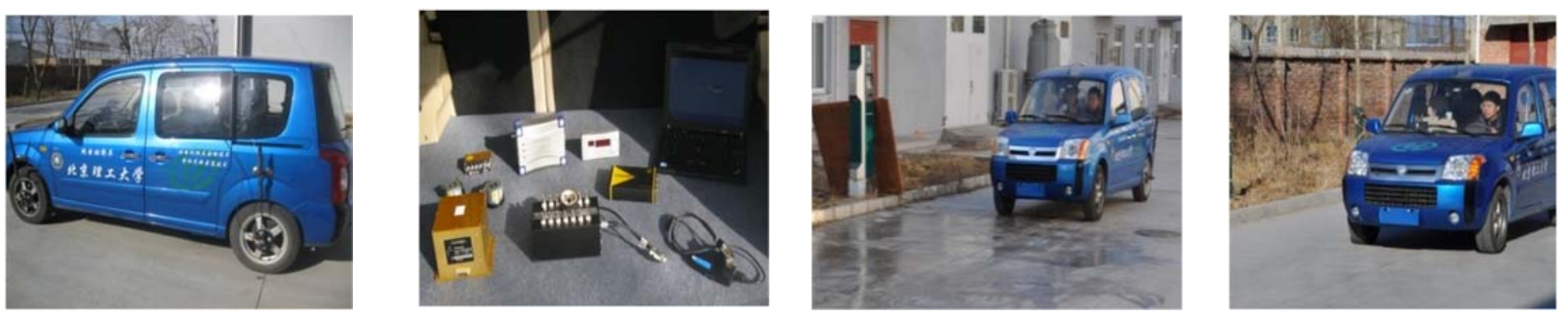

Figure.3 Research instrument and experimental road

\section{Estimation result}

Figure (4) show the measurement parameters obtained from the sensor. Figure (5) show the estimation result of the vehicle state vectors. Compared with the actual value, we can conclude that these vectors are well estimated. The estimation error is very small; this enables us to conclude that the estimation results are good. The tire slipping rate-friction coefficient curves are show in Figure (6): (a) for dry asphalt road and (b) for ice road. These diagrams are drawn based on the experimental measured samples.
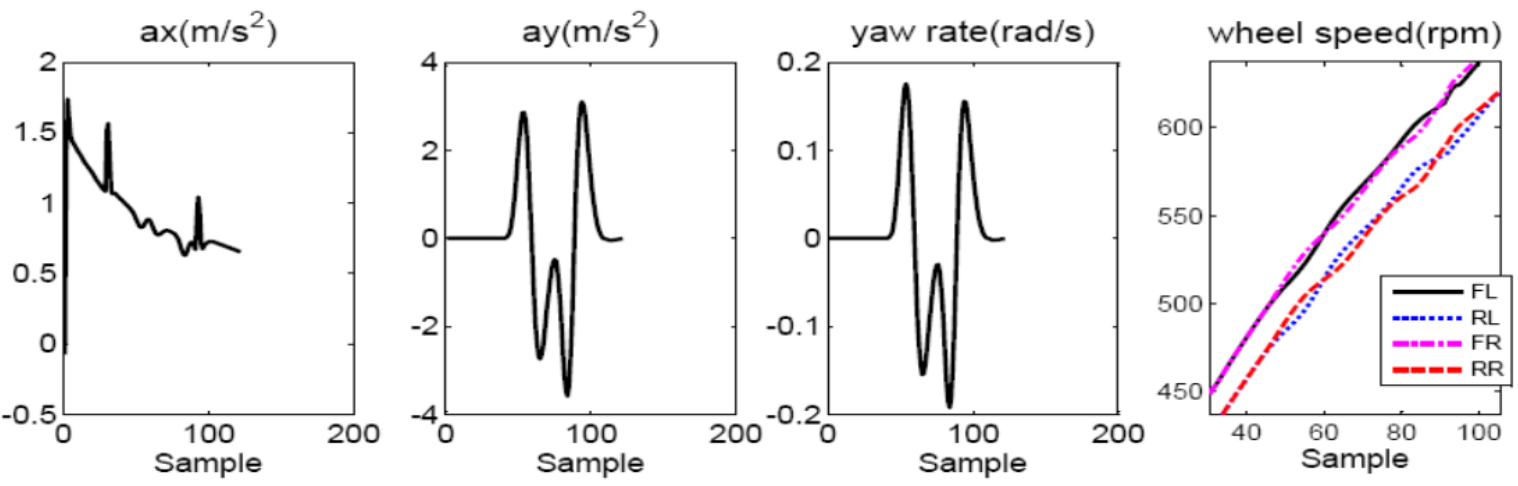

Figure.4 Measured parameters 

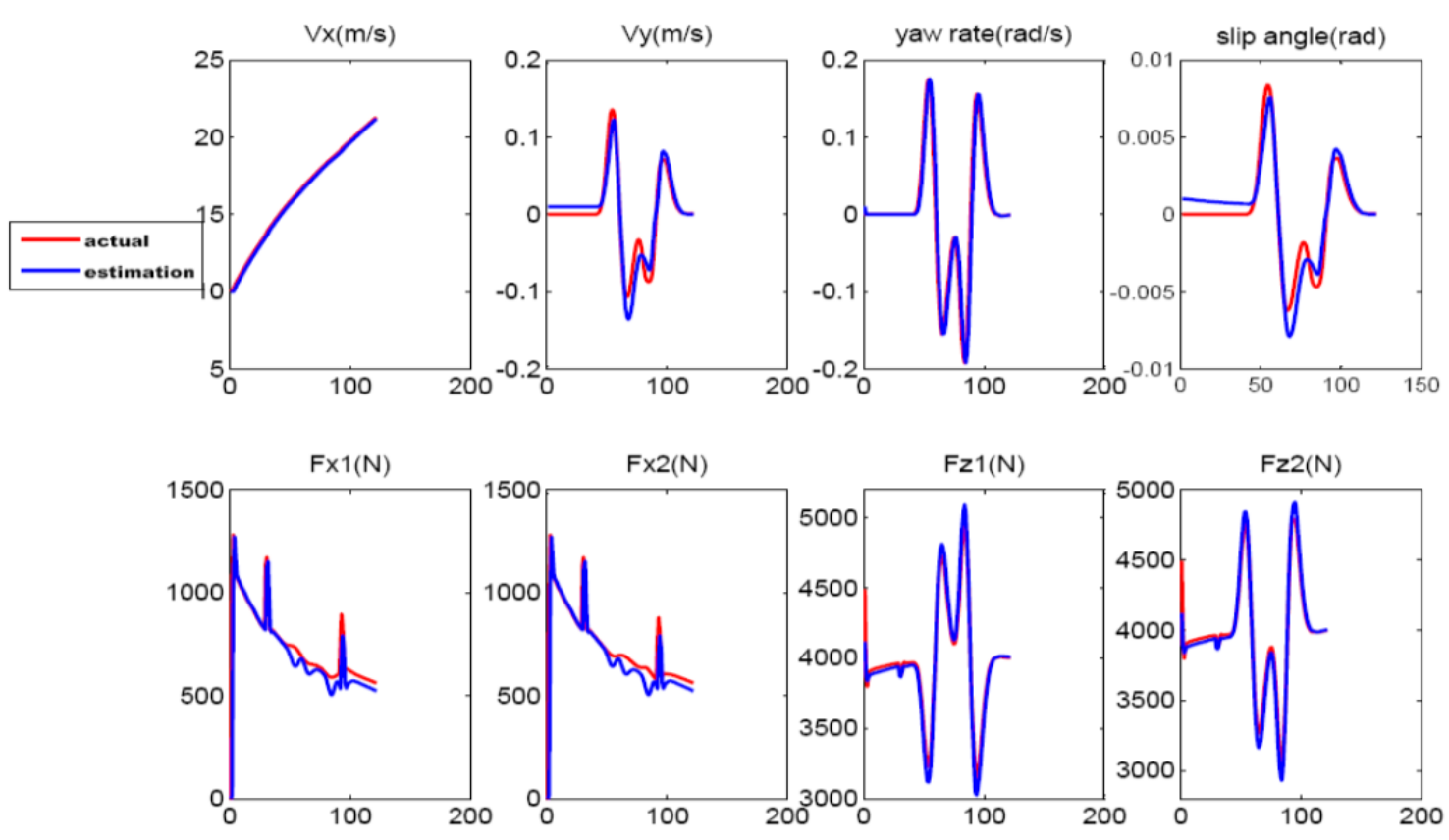

Figure.5 Estimation and actual vehicle states

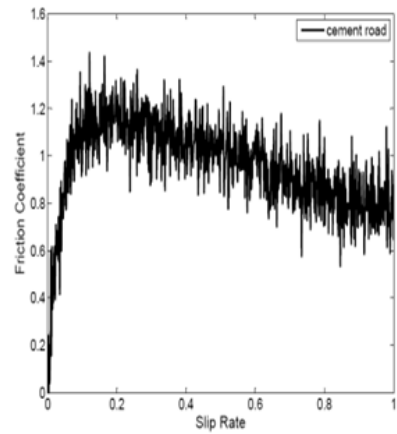

(a) dry asphalt road

Figure.6 Test data of the friction coefficient

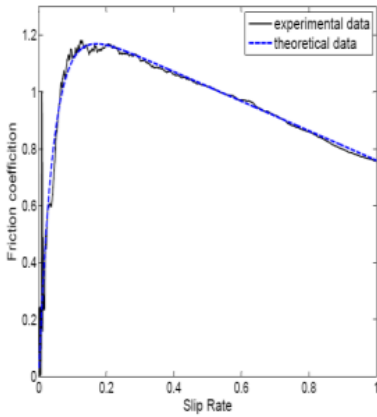

(a) dry asphalt road

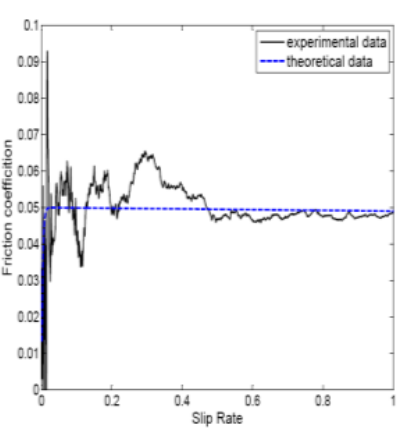

(b) ice road

Figure.7 Curve fitting results with RLS

\section{Conclusions}

This paper develops a real-time estimation of vehicle state and road surface based on the distributed driven electric vehicle. The extended Kalman filter and RLS algorithm were used in estimator design. The estimation results have been compared to real measurements collected with sensors. The estimation result of vehicle dynamic vector obtained by use the EKF observer. One can conclude that these vectors are well estimated. The method based on recursive least square with forgetting factor is used for road identification. This research shows that the algorithm is able to identification two surfaces: dry asphalt and ice. The proposed approach has several advantages such as accurate, effective, short cycle and low cost. Also, they have the ability to provide with reliable information for vehicle active safety control.

\section{Acknowledgment}

Project supported by the National Natural Science Foundation of China (Grant No.51175043) and the Foundation of Beijing Municipal Engineering Research Center of Electric Vehicles.

\section{References}

[1] Thomas D.Gillespie. Fundamental of Vehicle Dynamics [M]. Michigan: Society of Automotive Engineers. 2000:39-41. 
[2] Yu Zhisheng. Automobile Theory [M]. Beijing: China Machine Press,2006:90-93.

[3] H.S. Bae, J. Ryu, and J. Gerdes. Road grade and vehicle parameter estimation for longitudinal control using GPS. Proceedings of IEEE Conference on Intelligent Transportation Systems, 2001.

[4] M. Tanelli L. Piroddi1 S.M. Savaresi. Real-time identification of tire-road friction conditions[J]. IET Control Theory Appl., 2009, Vol. 3, Iss. 7, pp. 891-906.

[5] Y. Hori, Future vehicle driven by electricity and control-research on four-wheel-motored "UOT Electric March II, IEEE Trans. Ind. Electron., vol. 51, no. 5, 2004: 654-962.

[6] U. Kiencke and L. Nielsen, Automotive Control Systems For Engine, Driveline, and Vehicle. Springer-Verlag, 2005.

[7] Wenzel, T.A., Burnham, K.J., Blundell, M.V., Williams, R.A.,2006. Dual extended Kalman filter for vehicle state and parameter estimation. Vehicle System Dynamics, 44(2): 153-171.

[8] R. Rajamani, Vehicle Dynamics and Control[M]. New York: Springer-Verlag, 2005:99-108.

[9] M. SATRIA, M. C. BEST, State estimation of vehicle handling dynamics using non-linear robust extended adaptive Kalman filter, Vehicle System Dynamics, Vol.41, 2004, pp. 103-112.

[10]YI J., ALVAREZ L., HOROWITZ R.: 'Adaptive emergency braking control with underestimation of friction coefficient', Trans. Control Syst. Technol., 2002, 10, (3), pp. 381-392. 